\title{
Genetic variation of the striped hamster (Cricetulus barabensis) and the impact of population density and environmental factors
}

\author{
Hui-liang Xue, Jin-hui Xu, Lei Chen and Lai-xiang $\mathrm{Xu}^{*}$
}

\begin{abstract}
Background: The striped hamster (Cricetulus barabensis) is a dominant rodent species in the North China Plain and has suffered a continuous decline of population size in the last 17 years. However, little is known about the population genetic structure of this species and how it is influenced by geographical and environmental factors. In the present study, we investigated the genetic diversity and genetic differentiation of the striped hamsters in four geographical populations of different environmental features. The genetic variability for a sample of 158 animals from the four populations was estimated using data from 10 microsatellite loci.
\end{abstract}

Results: Genetic diversity exists in the striped hamster. The number of alleles and the proportion of heterozygosity indicate moderate variability. A total of 46 alleles were detected, with a moderate mean number of alleles. Significant genetic differentiation $(p<0.01)$ exists among all four examined geographical populations. Population-specific alleles and variable allelic richness were detected in all populations regardless of the geographical distances. The genetic diversity positively correlates with the population density, while the genetic distance is partially affected by the geographical distances. Moreover, the habitat environment is also an important factor influencing the genetic differentiation of the species.

Conclusions: The genetic diversity of the striped hamster is mainly impacted by population density, while partially influenced by geographical distances. The results of our study and other studies collectively suggest that a minimum geographical distance is required to prevent gene flow between different populations and for genetic differentiation to be pronounced.

Keywords: Population genetic structure; Genetic diversity; Genetic differentiation; The striped hamster; Population density; Microsatellite

\section{Background}

Population dynamics is a central issue in population ecology and varies greatly as a result of the interplay of genetic factors and environmental factors. Anthropogenic activities have imposed increasing impact on habitats and ecosystems of wildlife, and consequently, many species are suffering from decreasing population sizes (Liu et al. 2013). Genetic diversity is a key evolutionary variable that correlates with population size and is believed to be critical for population viability and the persistence of species. It is affected by a number of evolutionary

\footnotetext{
* Correspondence: xulx@mail.gfnu.edu

College of Life Sciences, Qufu Normal University, 57 Jingxuan West Road, Qufu, Shandong 273165, China
}

processes such as selection, gene flow, genetic drift, and dispersal patterns. Loss of genetic diversity of a population may result in declined fitness and the ability to persist and adapt in ever-changing environments (Garant et al. 2007; Lenormand 2002). Therefore, maintenance of genetic diversity is fundamental for wildlife conservation and management, and analysis of population genetic structure and the influencing factors in wild populations is the first step.

The striped hamster (Cricetulus barabensis), also known as the striped dwarf hamster, is one of the dominant rodent species in the North China Plain. It is also distributed in Russia, Mongolia, and Korea. The hamsters feed on stems and leaves of plant during summer and on foraging crop seeds in winter (Zhao et al. 2013). They are solitary and very aggressive and have been widely used as 
laboratory animals. Though considered a pest, the species has experienced a continued decline of the population density in the last 17 years, which is attributed mainly to agricultural activities (Yan et al. 2013). Various approaches have been used to forecast the population dynamics of the striped hamster (Wu et al. 2007a, b). Most research, however, have focused on the environmental and anthropogenetic factors (Hou et al. 1993; Song and Wang 2003; Yan et al. 2013), while the population genetic structure of the striped hamster is poorly understood. The application of mixed methods of evaluating patterns of individual genotypes, allele frequencies, and genetic structure is becoming a powerful means for descriptive genetic analysis. Such methods are being increasingly used for inference of population structure at the landscape scale, as is needed to forecast population dynamics. Effective strategies to forecast and control the abundance of the striped hamster incorporate an understanding of their present genetic structure and the distribution of genetic diversity throughout the geographical range. To date, however, there have been few such efforts to forecast population dynamics from patterns of genetic variation in the striped hamster (Xu et al. 2008). Large-scale studies of the population genetics of the striped hamster have not been carried out.

Polymorphic microsatellite loci are the most frequently used and the most informative molecular markers for analysis of genetic diversity and genetic differentiation (Kim and Sappington 2013). In the striped hamster, 14 microsatellite markers have been isolated and characterized (Xu et al. 2008). In the study, the authors analyzed the genetic diversity of the striped hamsters and detected no significant allelic variations among different populations of the striped hamster. The lack of genetic diversity may be explained by the short distance among the sampling locations $(60 \mathrm{~km})$ where no geographical barrier exists to prevent gene flow. Until now, no factors influencing genetic variation have been analyzed.

In the present study, by examining ten microsatellite loci, we investigated the population genetic structure of the striped hamsters from four geographical locations that are 167 to $945 \mathrm{~km}$ apart and of different environmental features. The four populations were from Wucun $(\mathrm{W})$, Pingyi $(\mathrm{P})$, Xilinhaote $(\mathrm{X})$, and Zhenglanqi (Zh) Counties of the North China Plain. We aimed (1) to reveal the genetic diversity of the striped hamsters in different geographical locations, (2) to examine the genetic differentiation among the geographical populations, (3) to analyze the relationship between the geographical and genetic distance, and (4) to compare the correlation between the genetic diversity and population density of the striped hamster to provide a basis for the conservation and management of population dynamics.

\section{Methods}

\section{Ethics statement}

All hamster procedures were reviewed and approved by the Institutional Animal Care and Use Committee of the Institute of Zoology, Chinese Academy of Sciences (Permit Number: IOZ11012) and by the Ethics Committee of Qufu Normal University. All researchers and students had received appropriate training and certification before performing animal studies.

\section{Sample collection and DNA extraction}

We captured striped hamsters using live traps made of wire mesh in the grassland areas of W, P, and X Counties and in the sandy area of Zh County of the North China Plain at the autumn of 2007. Previous studies suggest the need for sample sizes of at least 20 individuals to obtain accurate estimates of genetic distance (Kalinowski 2004). The sample size of each population in this study meets its requirements. One hundred and fifty-eight individuals were analyzed in this study, comprising 42 from W, 44 from P, 38 from $\mathrm{X}$, and 34 from $\mathrm{Zh}$. The rates of live traps, which represent population density (Yan et al. 2013), were $4.8 \%, 5.2 \%, 7.1 \%$, and $1.2 \%$ in $\mathrm{W}, \mathrm{P}, \mathrm{X}$, and Zh populations, respectively. The sampling locations in the present study are shown in Figure 1.

An approximate volume of $0.1 \mathrm{~mL}$ blood was taken from the lateral tail vein of each individual and preserved in 95\% ethanol. Whole genomic DNA was extracted using standard phenol-chloroform protocols and preserved in 95\% ethanol (Sambrook and Russell 2006). In order to prevent contamination during DNA extraction, benches and plasticware were cleaned with $10 \%$ bleach and sterile water and then exposed to ultraviolet (UV) light for $30 \mathrm{~min}$. The surface of muscle samples was also exposed to UV light for $30 \mathrm{~min}$. DNA samples were stored at $-20^{\circ} \mathrm{C}$ for further microsatellite genotyping. We used ten extraction controls, and none produced positive amplification during subsequent polymerase chain reaction (PCR).

\section{Microsatellite genotyping}

Ten microsatellite loci for C. barabensis (Song et al. 2011; $\mathrm{Xu}$ et al. 2008) were used for this study on the basis of their efficiency. Variation at each locus was assessed by PCR. Amplification reactions were carried out in a total volume of $25 \mu \mathrm{L}$ containing $2.5 \mu \mathrm{L}$ of $10 \times$ PCR buffer, 2.5 $\mathrm{mM} \mathrm{MgCl}_{2}, 0.2 \mathrm{nM}$ dNTP, 1 U Taq DNA polymerase (Promega Corporation, Fitchburg, WI, USA), 10 pM of forward and reverse primers, and approximately 2 ng of template DNA using the following temperature profile: 5 min at $94^{\circ} \mathrm{C}$; followed by 30 cycles of $45 \mathrm{~s}$ at $94^{\circ} \mathrm{C}, 45 \mathrm{~s}$ at $46^{\circ} \mathrm{C}$ to $58^{\circ} \mathrm{C}$, and $30 \mathrm{~s}$ at $72^{\circ} \mathrm{C}$; followed by a final extension of $10 \mathrm{~min}$ at $72^{\circ} \mathrm{C}$; and storage at $4^{\circ} \mathrm{C}$. After amplification, PCR products were diluted and genotyped on a 


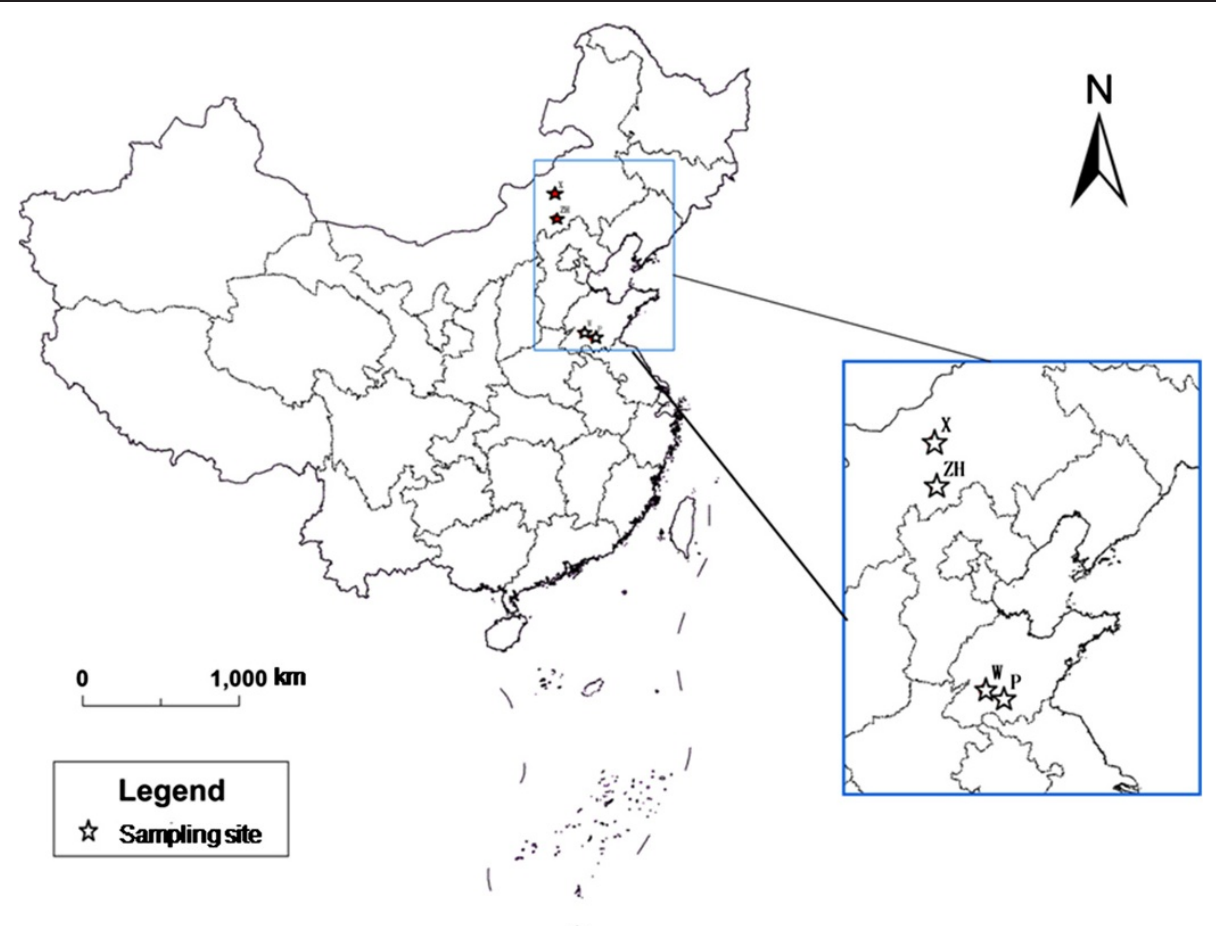

Figure 1 Map of sampling locations of the present study. The four sampling sites are Wucun (W), Pingyi (P), Xilinhaote (X), and Zhenglanqi (Zh) Counties of the North China Plain. W and P are in Shandong province, while $X$ and Zh are in Inner Mongolia.

Beckman CEQ8000 (Beckman Coulter, Inc., Brea, CA, USA) according to the manufacturer's instructions using CEQ8000 software and GeneMarker version 1.5 (SoftGenetics, State College, PA, USA).

\section{Data analysis}

Analysis of molecular variance (AMOVA) was used to examine the diversity of the striped hamster within and among the populations using a microsatellite marker. AMOVA tests were hierarchical and used 1,000 permutations as implemented in Arlequin to test whether each of the two levels of organization (within and among populations) explained a significant portion of the overall microsatellite diversity.

Diversity was analyzed per locus and per population on the basis of allelic richness $\left(R_{S}\right.$, number of alleles independent of sample size), observed $\left(H_{O}\right)$ and expected $\left(H_{E}\right)$ heterozygosity, and linkage disequilibrium. All calculations were performed in FSTAT (Goudet 1995) with significance based on Bonferroni-corrected $P$ values after 10,000 random permutations. Deviations from HardyWeinberg equilibrium (HWE) for each population were assessed using an exact test implemented in GENEPOP (Raymond and Rousset 1995).

To compare population-level relationships identified by microsatellite markers, we constructed neighbor-joining (NJ) dendrograms based on pairwise genetic distances. NJ dendrograms for microsatellites were constructed in NEIGHBOR and PHYLIP version 3.5 (Felsenstein 1993). Support for microsatellite Nei's distance dendrogram was based on 1,000 replicate distance measures constructed in MSA by permuting genotypes among populations (Dieringer and Schlotterer 2003).

Genetic differentiation between geographical populations was assessed by comparing the average number of pairwise differences between different populations (PiXY), the average number of pairwise differences within populations ( $\mathrm{PiX}$ and $\mathrm{PiY}$ ), and the corrected average pairwise difference $(\mathrm{PiXY}-(\mathrm{PiX}+\mathrm{PiY}) / 2)$ using Arlequin 2.000 .

\section{Results}

\section{Microsatellite polymorphisms}

A total of 46 alleles were detected using the 10 microsatellite loci from 158 individuals (W 42, P 44, X 38, Zh 4), with a mean allele number per locus of 4.6 (range 1.70 in $\mathrm{Zh}$ to 2.30 in $\mathrm{X}$ ). While some alleles were more restricted, the others showed a wide geographical distribution (shown in Additional file 1: Table S1). Twenty-one of 46 alleles were found in only a single population. Among the ten loci, the number of alleles observed per locus varied from two (EF543185) to six (DQ459487, DQ459492, DQ459494, EF543179, EF543177, and EF543186), as shown in Additional file 1: Table S2. 
Table 1 Average gene diversity over loci based on the number of different alleles

\begin{tabular}{lllll}
\hline & \multicolumn{3}{l}{ Populations } & \\
\cline { 2 - 5 } & $\mathbf{W}$ & $\mathbf{P}$ & $\mathbf{X}$ & $\mathbf{Z h}$ \\
\hline Gene diversity & $0.40 \pm 0.23$ & $0.43 \pm 0.24$ & $0.52 \pm 0.28$ & $0.29 \pm 0.17$ \\
Trap success, \% & 4.8 & 5.2 & 7.1 & 1.2 \\
\hline
\end{tabular}

Estimates of allelic richness $\left(R_{S}\right)$, observed $\left(H_{O}\right)$ heterozygosity, and expected $\left(H_{E}\right)$ heterozygosity for the microsatellites in each population are shown in Additional file 1: Table S3. Allelic richness in the $\mathrm{X}$ population was higher and ranged from 3.8 to 4.9 alleles per locus (average $=4.1$ ). Polymorphism was moderate in $\mathrm{W}$ and $\mathrm{P}$ populations with allelic richness ranging from 1.0 to 3.7 alleles per locus (average $=2.7$ ) and from 1.0 to 4.0 alleles per locus (average $=2.7$ ), respectively. In the $\mathrm{Zh}$ population, average allelic richness (average $=1.6$ ) per sample over all loci was lower compared to that in the other examined populations.

Observed heterozygosity ranged from 0 to 0.81 (average = 0.35 ), with the lowest average value (average $=0.25$ ) for the $\mathrm{Zh}$ population and the highest average value (average $=$ $0.44)$ for the $X$ population. Expected heterozygosity ranged from 0 to 0.67 , with the lowest average value (average $=$ 0.29 ) for the $\mathrm{Zh}$ population and the highest average value (average $=0.52$ ) for the $\mathrm{X}$ population.

After Bonferroni correction, all ten loci significantly deviated from HWE in the X population, EF543178 loci in the W population, and EF48900 loci in the P population. All ten loci were within HWE in the Zh population.

Diversity indices, average gene diversity over loci, are summarized in Table 1. Higher genetic diversity (0.52) was found in the $\mathrm{X}$ population while moderate in $\mathrm{W}$ (0.40) and $\mathrm{P}(0.43)$ populations and lower in Zh (0.29) population. The genetic diversity was positively related to the rate of live traps (Figure 2), which represent the population densities.

\section{Genetic differentiation}

Allele distribution displays certain geographical specificity (Additional file 1: Table S2). Allele 330 at DQ459487 loci, allele 309 at DQ459492 loci, allele 391 at EF543179 loci, allele 380 at EF543177 loci, allele 182 at EF543178 loci, and allele 348 at EF530613 loci were detected only in the $\mathrm{W}$ population. Population $\mathrm{P}$ also possesses the following specific alleles: 315 at DQ459492 loci, 404 and 484 at EF543179 loci, 392 and 450 at EF543177 loci, and 400 at EF543186 loci. The specific alleles that only exist in the X population were 335 at DQ459487 loci, 390 and 406 at DQ459494 loci, and 280 and 330 at EF543177 loci. Allele 345 at DQ459487, allele 330 at DQ459492, allele 406 at EF543179, and allele 200 at EF543186 were specific alleles in the Zh population. Meanwhile, the tested populations possess some common alleles shown in Additional file 1: Table S2.

The test of population differentiation showed that these four geographical populations are significantly differentiated from each other in pairwise comparisons $(p<0.001)$ (Table 2).

\section{Analyses of population genetic structure}

Genetic and geographical distances among the examined populations are summarized in Table 3. A higher genetic distance was found between $\mathrm{Zh}$ and $\mathrm{W}$ (2.35) and $\mathrm{Zh}$ and $\mathrm{P}$ (1.68) populations. The genetic distance between $\mathrm{X}$ and $\mathrm{Zh}$ populations was moderate (0.74). The genetic distances among $\mathrm{W}, \mathrm{P}$, and $\mathrm{X}$ populations were lower (0.62 to 0.63 ). The geographical distances, based on the electronic map, are also listed in Table 3. The correlation

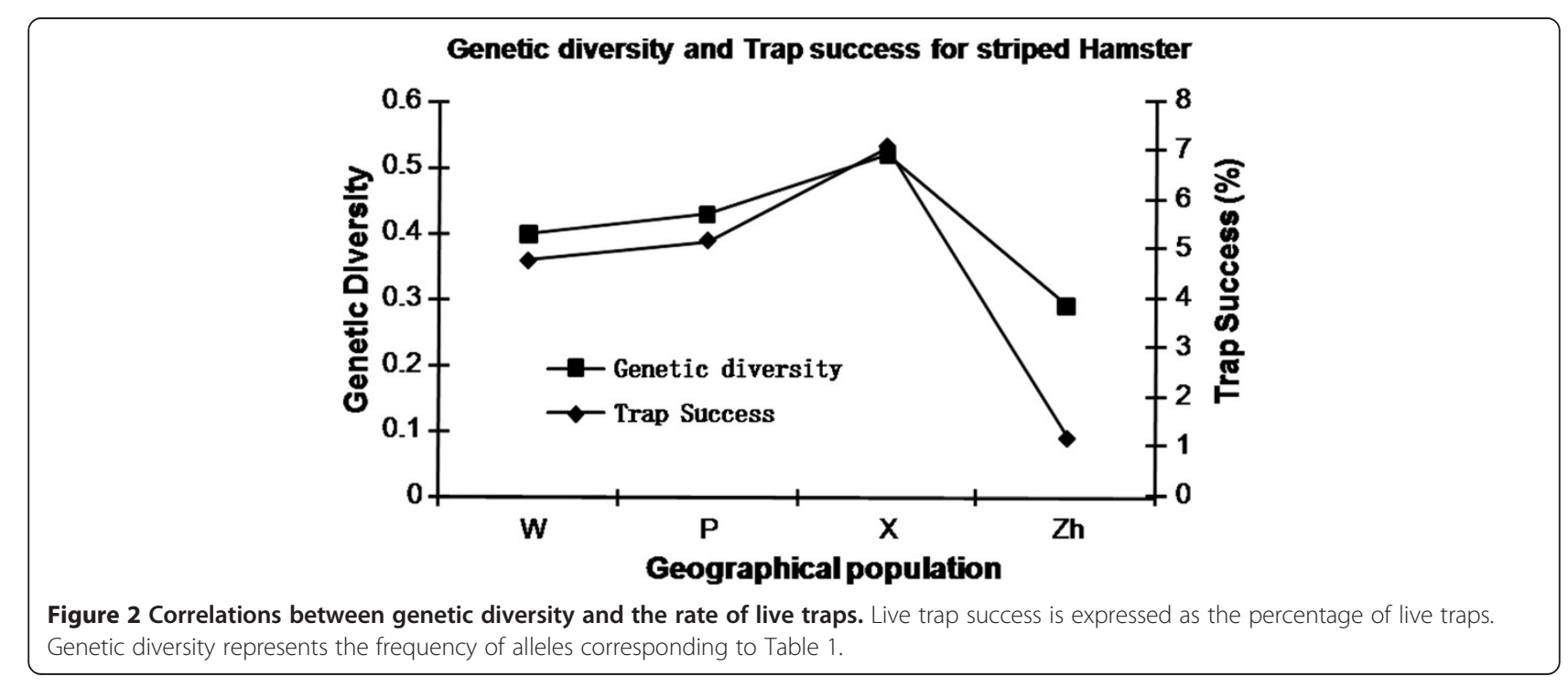


Table 2 Pairwise genetic differentiation for microsatellite data of the striped hamster based on Nei's distance method

\begin{tabular}{lllll}
\hline Populations & W & $\mathbf{P}$ & $\mathbf{X}$ & Zh \\
\hline W & 4.04 & $6.84(p=0.000)$ & $7.04(p=0.000)$ & $9.48(p=0.000)$ \\
P & $2.68(p=0.001)$ & 4.28 & $7.27(p=0.000)$ & $8.81(p=0.000)$ \\
X & $2.40(p=0.001)$ & $2.51(p=0.001)$ & 5.23 & $7.10(p=0.000)$ \\
Zh & $6.01(p=0.000)$ & $5.22(p=0.000)$ & $3.03(p=0.001)$ & 2.89 \\
\hline
\end{tabular}

Above diagonal: average number of pairwise differences between populations (PiXY). Diagonal elements: average number of pairwise differences within populations (PiX). Below diagonal: corrected average pairwise difference $[\mathrm{PiXY}-(\mathrm{PiX}+\mathrm{PiY}) / 2]$. Corrected average pairwise differences, which are statistically different, are indicated.

between the genetic and geographical distances is shown in Figure 3.

NJ analysis of populations based on standard genetic distance, firstly, produced two groups: A (W, P, and X populations) and B (Zh population). Secondly, A group could be subdivided into two subgroups: 1 (X) and 2 (W and P) as shown in Figure 4.

relationship between genetic diversity and the influencing factors, including population density, geographical distance, and environmental factors, is discussed below.

\section{Discussion}

\section{Genetic diversity and genetic differentiation}

Geographical variation in population genetic structure across species' ranges is critical for the environmental adaptation and evolution of the species. The importance of genetic diversity for the viability of populations is widely accepted (Frankham et al. 2010; Hansson and Westerberg 2002; Reed and Frankham 2003; Schmitt and Hewitt 2004). Genetic diversity and differentiation of populations are dictated by the interplay of genetic drift, gene flow, and natural selection. Variation in population turnover and population size may have a major impact on these processes. Gene flow, determined by the mobility of the species and geographical distance, promotes genetic diversity and precludes differentiation, while selection and drift enhance genetic differentiation if populations are sufficiently isolated. As the isolation of population increases and the population size reduces, genetic drift and inbreeding depression become more influential than gene flow, and the differentiation among populations is expected to become greater and more variable. Greater genetic diversity, represented by allele

Table 3 Geographical and genetic distances among different populations

\begin{tabular}{lllll}
\hline Populations & W & $\mathbf{P}$ & $\mathbf{X}$ & $\mathbf{Z h}$ \\
\hline W & & 0.63 & 0.63 & 2.35 \\
P & 167 & & 0.62 & 1.68 \\
X & 931 & 945 & & 0.74 \\
Zh & 748 & 766 & 185 & \\
\hline
\end{tabular}

Above diagonal: genetic distance. Below diagonal: geographical distance $(\mathrm{km})$. richness and degree of heterozygosity, correlates significantly with fitness.

Among the examined geographical populations in our study, there exist significant genetic differences. Populationspecific alleles and variable allelic richness were detected in all populations regardless of the geographical distances. This may be attributed to the species' relatively restricted mobility and habitat availability in the local area, whereby selection and genetic drift may be important factors influencing population genetic characters. In another report (Xu et al. 2008), no significant differences in genetic variation were detected among the three striped hamster populations that were only $60 \mathrm{~km}$ apart. In our study, the shortest distance between two populations is $185 \mathrm{~km}$, which is much greater than that in the study by $\mathrm{Xu}$ et al. Thus, it appears that a minimum distance (between 60 and $185 \mathrm{~km}$ ) between two populations is a prerequisite for genetic differentiation to be pronounced.

Two commonly used indices of genetic diversity, allelic richness, and heterozygosity (observed and expected heterozygosity, $H_{O}$ and $H_{E}$, respectively) for the microsatellites in the examined populations are in the same order of $\mathrm{X}>\mathrm{P}>\mathrm{W}>\mathrm{Zh}$ populations. Correspondingly, the density of the populations was in the similar sequence of $\mathrm{X}>\mathrm{P}>\mathrm{W}>\mathrm{Zh}$ populations. As shown in Figure 2, an obvious correlation can be inferred between the levels of genetic diversity and the population density. Our results are in accordance with studies in other species in which a similar correlation was found (Aspi et al. 2006; Berthier et al. 2006; Eckert et al. 2008; Frankham 1996; Furlan et al. 2012; Montgomery et al. 2000; Wang et al. 2013), including other rodents (Krebs 2013) and hamster species (Romanenko et al. 2007), like the greater longtailed hamster (Dong et al. 2010; Xie and Zhang 2006; Xu et al. 2013a; Xue et al. 2014), the golden hamster (Karsten et al. 2005), and the common hamster (Banaszek et al. 2012; Neumann et al. 2005). Similarly, the genetic diversity of the striped hamster has experienced a trend of continuous decline in recent years. However, the genetic distance of the striped hamster correlates partially with geographical distance, while it is more greatly influenced by environmental factors. Strong fluctuations in population density can lead to loss of genetic diversity, and 


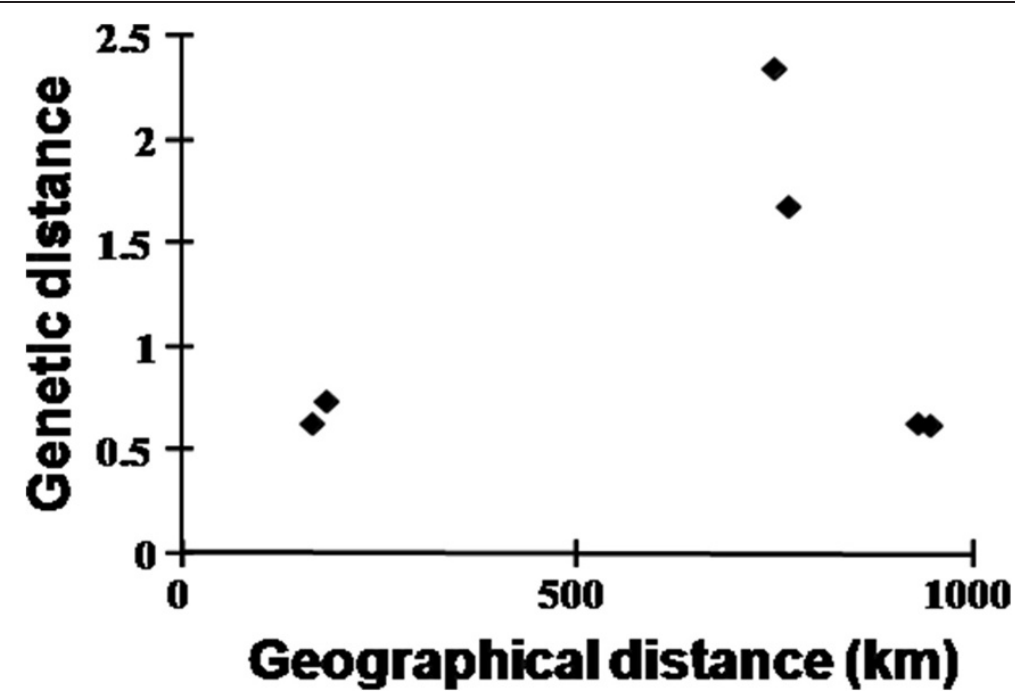

Figure 3 Correlations between genetic distance (measured as Nei's (1978) genetic distance) and geographical distance. Genetic distance values are corresponding to those in Table 3.

in return, reduction in genetic diversity and inbreeding depression can significantly lower the ability of populations to adapt to changing environments, eventually leading to extinction (Frankham 1996). Therefore, maintenance of genetic diversity is an essential issue of conservation.

\section{Population structure}

Microsatellite DNA data for the striped hamster revealed significant genetic polarization among populations, with deep genetic divergence between the two lineages (A and B), as shown in Figure 4. The examined populations based on geographical distance could be divided into clusters I (W and P) and II (X and Zh), which was in discrepancy with the genetic analysis and implying that genetic distance was not positively correlated with geographical distance (Figure 3). The common character of the populations in lineage A is that their habitat environments were all grassland areas, while the habitat environment of the $\mathrm{Zh}$ population in lineage A was a sandy area.
The populations based on its habitat environments could be partitioned into two lineages: $a(X, W$, and $\mathrm{P})$ and $\mathrm{b}$ (Zh), which was in accordance with the results of genetic analysis and implying that habitat environment is an important factor influencing its genetic differentiation. A previous study showed that different living condition is a reason leading to genetic differentiation among populations (Wang et al. 2008), which was in accordance with the results of this study.

The lineage A based on genetic distance could be subdivided into two subgroups: $1(\mathrm{X})$ and 2 (W and P populations), which was in accordance with the result analyzed by the geographical distance. Therefore, isolation by distance (IBD) may partly account for the genetic differentiation among $\mathrm{W}, \mathrm{P}$, and $\mathrm{X}$ populations, which was in accordance with the results of former scientists (Jiang et al. 2007; Xu et al. 2013b; Zi-Yan 2008).

That lineage B (population Zh) is greatly differentiated from the other populations raises the possibility to classify the $\mathrm{Zh}$ population as a subspecies. Currently, subspecies

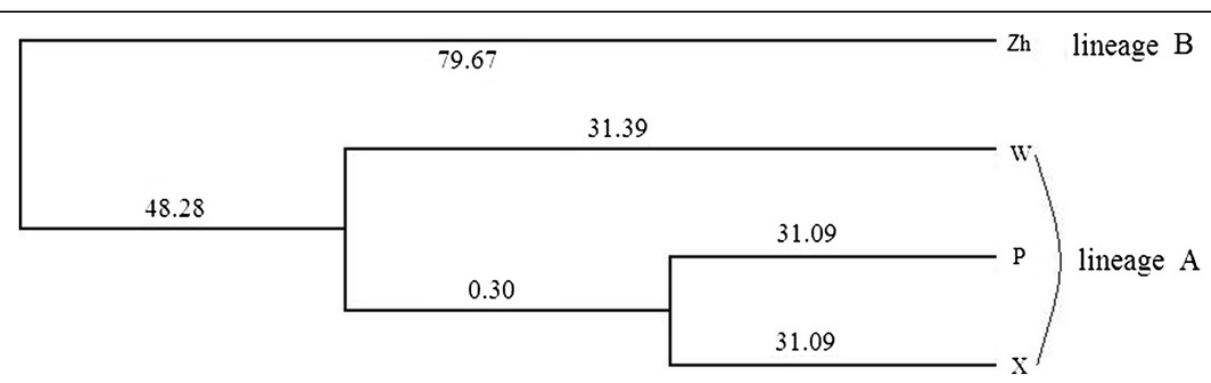

Figure 4 NJ tree based on Nei's (1978) genetic distance by the method. Modified from the NEIGHBOR procedure of PHYLIP version 3.5. All populations are marked. Bootstrap values are shown. 
are defined by a combination of morphological, behavioral, and genetic characters, among which phylogenetic distinction is considered to be essential (Haig et al. 2006). However, these criteria have not been applied to hamsters, and a standard of how large the genetic distance to define a hamster subspecies is lacking. We have not observed any morphological or behavioral differences between the $\mathrm{Zh}$ population and other populations. In light of the phylogenetics to define subspecies in other species, such as birds (Phillimore and Owens 2006), the genetic distance of lineage $\mathrm{B}$ is large enough to define the $\mathrm{Zh}$ population as a subspecies. Intriguingly, the geographical distance between $\mathrm{Zh}$ and $\mathrm{X}$ populations appears to be close enough for genetic interactions and no barrier exists to prevent gene flow, yet they show distinct phylogenetic patterns. The highest differentiation and lowest genetic diversity that correlate with the lowest population density are implicative of inbreeding depression within the $\mathrm{Zh}$ population. We believe that a more extensive sampling, including regions of Mongolia and Korea, should provide a more complete picture of the morphological, behavioral, and phylogenetic patterns of the striped hamster and to precisely define subspecies.

The genetic differentiation within the breed resulted from the interactions of genetic and environmental factors. Gene flow belongs to genetic factors, which is variably affected by geographical distance, habitat fragmentation, and so on. Habitat environment belongs to environmental factors such as grassland and sandy areas, which plays an important role in selection. Genetic diversity and ecological factors can have reciprocal influences (Hughes et al. 2008). Genetic diversity impacts significantly on ecological processes such as primary productivity, population recovery from disturbance, interspecific competition, community structure, and fluxes of energy and nutrients. In return, ecological and environmental factors can also play a role in shaping genetic diversity patterns (Gaggiotti et al. 2009). Based on the above analysis, genetic differentiation within the striped hamster was caused by the interaction of genetic and environmental factors, in which environmental factors play a more important role.

\section{Conclusions}

We have, for the first time, systematically analyzed the population genetic structure of the striped hamsters and the influencing factors. Our data revealed that there is a moderate level of overall genetic diversity (allele richness and heterozygosity) and significantly different genetic variation among the studied populations. The genetic diversity is positively correlated to the population density and is greatly affected by the environmental factors, while geographical isolation by distance contributes partially to the genetic differentiation among the different populations. In addition, the results of our study and other studies collectively suggest that a minimum geographical distance (between 60 and $185 \mathrm{~km}$ ) is required for genetic differentiation to be pronounced. Our data provide the first empirical evidence for the existence of genetic differentiation and the influencing factors in the striped hamster.

\section{Additional file}

Additional file 1: Table S1. Genotypes of ten microsatellite loci for the
four examined geographical populations of striped hamster. Table S2.
Alleles examined in the striped hamster at ten microsatellite markers for
different geographical populations. Table S3. Allelic richness, observed
heterozygosity, and expected heterozygosity at ten microsatellite markers
for different striped hamster populations.

Competing interests

The authors declare that they have no competing interests.

\section{Authors' contributions}

$H L X$ and LXX conceived the study. HLX designed the study. JHX performed the experiments and acquired the data. LC analyzed the data. $L C$ and HLX performed the statistical analysis. $H L X$ and $J H X$ prepared the manuscript. $L X X$ and LC edited the manuscript. LXX reviewed the manuscript and guaranteed the integrity of the entire study. All authors read and approved the final manuscript.

\section{Acknowledgements}

The authors are grateful to Dr. Wan from the Institute of Zoology, Chinese Academy of Science for providing samples from $\mathrm{X}$ and $\mathrm{ZH}$. This work was supported by the National Natural Science Foundation of China (31270417, 31300304).

Received: 5 May 2014 Accepted: 2 September 2014

Published online: 19 September 2014

\section{References}

Aspi J, Roininen E, Ruokonen M, Kojola I, Vila C (2006) Genetic diversity, population structure, effective population size and demographic history of the Finnish wolf population. Mol Ecol 15:1561-1576

Banaszek A, Ziomek J, Jadwiszczak KA, Kaczynska E, Mirski P (2012) Identification of the barrier to gene flow between phylogeographic lineages of the common hamster Cricetus cricetus. Acta Theriol (Warsz) 57:195-204

Berthier K, Charbonnel N, Galan M, Chaval Y, Cosson JF (2006) Migration and recovery of the genetic diversity during the increasing density phase in cyclic vole populations. Mol Ecol 15:2665-2676

Dieringer D, Schlotterer C (2003) Two distinct modes of microsatellite mutation processes: evidence from the complete genomic sequences of nine species. Genome Res 13:2242-2251

Dong J, Li C, Zhang Z (2010) Density-dependent genetic variation in dynamic populations of the greater long-tailed hamster (Tscherskia triton). J Mammal 91:200-207

Eckert CG, Samis KE, Lougheed SC (2008) Genetic variation across species' geographical ranges: the central-marginal hypothesis and beyond. Mol Ecol 17:1170-1188

Felsenstein F (1993) PHYLIP (Phylogeny Inference Package), Version 3.5c

Frankham R (1996) Relationship of genetic variation to population size in wildlife. Conservat Biol 10:1500-1508

Frankham R, Ballou JD, Briscoe DA (2010) Introduction to conservation genetics, 2nd edn. Cambridge University Press, Cambridge

Furlan E, Stoklosa J, Griffiths J, Gust N, Ellis R, Huggins RM, Weeks AR (2012) Small population size and extremely low levels of genetic diversity in island populations of the platypus, Ornithorhynchus anatinus. Ecol Evol 2:844-857

Gaggiotti OE, Bekkevold D, Jorgensen HB, Foll M, Carvalho GR, Andre C, Ruzzante DE (2009) Disentangling the effects of evolutionary, demographic, and environmental factors influencing genetic structure of natural populations: Atlantic herring as a case study. Evolution 63:2939-2951 
Garant D, Forde SE, Hendry AP (2007) The multifarious effects of dispersal and gene flow on contemporary adaptation. Funct Ecol 21:434-443

Goudet J (1995) Fstat (version 1.2): A computer program to calculate f-statistics. Journal of Heredity 86:485-486

Haig SM, Beever EA, Chambers SM, Draheim HM, Dugger BD, Dunham E-SE, Fontaine JB, Kesler DC, Knaus BJ, Lopes IF, Loschl P, Mullins TD, Sheffield LM (2006) Taxonomic considerations in listing subspecies under the U.S. Endangered Species Act. Conserv Biol 20:1584-1594

Hansson B, Westerberg L (2002) On the correlation between heterozygosity and fitness in natural populations. Mol Ecol 11:2467-2474

Hou X, Dong W, Yang Y, Zhou Y (1993) Studies on the population dynamics of striped hamster in Huhehot district. Zool Res 14:143-149

Hughes AR, Inouye BD, Johnson MTJ, Underwood N, Vellend M (2008) Ecological consequences of genetic diversity. Ecology Letters 11:609-623

Jiang Y-S, Liu W-Z, Zhang C-X, Qiao L-Y, Zhu W-J, Zhang G-X, Guo C-J (2007) AFLP analysis of genetic diversity of Apis cerana Fabricius distributed in different geographic areas in China. Acta Entomologica Sinica 50:144-152

Kalinowski ST (2004) Do polymorphic loci require large sample sizes to estimate genetic distances? Heredity 94:33-36

Karsten N, Steffen M, Peter F, Rolf G (2005) Microsatellites for diversity studies in the golden hamster. Mol Ecol Resour 5:876-878

Kim K, Sappington TW (2013) Microsatellite data analysis for population genetics. In: Kantartzi SK (ed) Microsatellites: Methods and Protocols. Methods in Molecular Biology. Humana Press, Springer Science+Business Media, LLC, New York, NY, pp 271-295

Krebs CJ (2013) Population fluctuations in rodents. University of Chicago Press, Chicago

Lenormand T (2002) Gene flow and the limits to natural selection. Trends Ecol Evol 17:183-189

Liu Y, Webber S, Bowgen K, Schmaltz L, Bradley K, Halvarsson P, Abdelgadir M, Griesser M (2013) Environmental factors influence both abundance and genetic diversity in a widespread bird species. Ecol Evol 3:4683-4695

Montgomery M, Woodworth L, Nurthen R, Gilligan D, Briscoe D, Frankham R (2000) Relationships between population size and loss of genetic diversity: comparisons of experimental results with theoretical predictions. Conservat Genet 1:33-43

Nei M (1978) Estimation of average heterozygosity and genetic distance from a small number of individuals. Genetics 89:583-590

Neumann K, Michaux JR, Maak S, Jansman HA, Kayser A, Mundt G, Gattermann R (2005) Genetic spatial structure of European common hamsters (Cricetus cricetus) — a result of repeated range expansion and demographic bottlenecks. Mol Ecol 14:1473-1483

Phillimore AB, Owens IP (2006) Are subspecies useful in evolutionary and conservation biology? Proc Biol Sci 273:1049-1053

Raymond M, Rousset F (1995) GENEPOP (version 1.2): population genetics software for exact tests and ecumenicism. J Heredity 86:248-249

Reed DH, Frankham R (2003) Correlation between fitness and genetic diversity correlación entre adaptabilidad y diversidad genética. Conservat Biol 17:230-237

Romanenko SA, Volobouev VT, Perelman PL, Lebedev VS, Serdukova NA, Trifonov VA, Biltueva LS, Nie W, O'Brien PC, Bulatova N, Ferguson-Smith MA, Yang F, Graphodatsky AS (2007) Karyotype evolution and phylogenetic relationships of hamsters (Cricetidae, Muroidea, Rodentia) inferred from chromosomal painting and banding comparison. Chromosome Res 15:283-297

Sambrook J, Russell DW (2006) The condensed protocols from molecular cloning: a laboratory manual. Cold Spring Harbor Laboratory Press, Cold Spring Harbor

Schmitt T, Hewitt GM (2004) The genetic pattern of population threat and loss: a case study of butterflies. Mol Ecol 13:21-31

Song Z-G, Wang D-H (2003) Metabolism and thermoregulation in the striped hamster Cricetulus barabensis. J Therm Biol 28:509-514

Song GH, Geng JN, Jia RY, Yue WB, Liu TF, Hu SN (2011) Isolation and characterization of 16 novel microsatellite loci in two inbred strains of the Chinese hamster (Cricetulus griseus). Genet Mol Res 10:2245-2256

Wang LP, Geng RQ, Chang H, Yang ZP, Sun W, Ji DJ, Wang QH (2008) Genetic differentiation of different geographical populations of indigenous goat in Jiangsu. Acta Agriculturae Boreali-occidentais Sinica 17:46-49, 53

Wang S, Zhu W, Gao X, Li X, Shaofei Y, Liu X, Yang J, Gao Z, Li Y (2013) Population size and time since island isolation determine genetic diversity loss in insular frog populations. Mol Ecol 23:637-648
Wu WH, Fu HP, Wu XD, Yang YP, Dong WH, Xu SL (2007a) Forecasting the population dynamics of Meriones unguiculatus and Cricetulus barabensis by applying Markov model. Chinese J Zoology 42:69-78

Wu WH, Fu HP, Wu XD, Dong WH, Xu SL (2007b) Forecasting the population dynamics of Cricetulus barabensis and Meriones unguiculatus by time series method. J Inner Mongolia Agric Univ 28:6-11

Xie J, Zhang Z (2006) Genetic diversity decreases as population density declines: implications of temporal variation in mitochondrial haplotype frequencies in a natural population of Tscherskia triton. Integr Zool 1:188-193

Xu L, Song M, Zhang F, Xu Y, Gao Q, Li C, Lu Y (2008) Isolation and characterization of microsatellite markers in the striped hamster (Cricetulus barabensis). Mol Ecol Resour 8:1500-1502

Xu L, Xue H, Song M, Zhao Q, Dong J, Liu J, Guo Y, Xu T, Cao X, Wang F, Wang S, Hao S, Yang H, Zhang Z (2013a) Variation of genetic diversity in a rapidly expanding population of the greater long-tailed hamster (Tscherskia triton) as revealed by microsatellites. PLoS One 8:e54171

Xu X, Zhou S, Zhu X, Zhou B (2013b) Microsatellite DNA analysis of genetic diversity of (Apis cerana cerana) in Hainan Island, southern China. Acta Entomologica Sinica 56:554-560

Xue H, Zhong M, Xu J, Xu L (2014) Geographic distance affects dispersal of the patchy distributed greater long-tailed hamster (Tscherskia triton). PLoS One 9:e99540

Yan C, Xu L, Xu T, Cao X, Wang F, Wang S, Hao S, Yang H, Zhang Z (2013) Agricultural irrigation mediates climatic effects and density dependence in population dynamics of Chinese striped hamster in North China Plain. J Anim Ecol 82:334-344

Zhao ZJ, Zhu QX, Chen KX, Wang YK, Cao J (2013) Energy budget, behavior and leptin in striped hamsters subjected to food restriction and refeeding. PLoS One 8:e54244

Zi-Yan N (2008) Genetic diversity of geographical populations of four Dendrolimus species (Lepidoptera: Lasiocampidae) in China based on allozyme analysis. Acta Entomologica Sinica 51:417-423

\section{doi:10.1186/s40555-014-0063-x}

Cite this article as: Xue et al:: Genetic variation of the striped hamster (Cricetulus barabensis) and the impact of population density and environmental factors. Zoological Studies 2014 53:63.

\section{Submit your manuscript to a SpringerOpen ${ }^{\circ}$ journal and benefit from:}

- Convenient online submission

- Rigorous peer review

- Immediate publication on acceptance

- Open access: articles freely available online

- High visibility within the field

- Retaining the copyright to your article

Submit your next manuscript at $>$ springeropen.com 